\title{
The effect of asymmetrical association on positive and negative semantic priming
}

\author{
KEITH A. HUTCHISON \\ State University of New York, Albany, New York
}

\begin{abstract}
One's actively ignoring a stimulus can impair subsequent responding to that stimulus. This negative priming effect has been argued to generalize to semantically related items as well, but the evidence for this is still somewhat weak. This article presents a new experiment in which participants made lexical decisions to asymmetrically associated prime-target pairs presented in either the forward (e.g., stork-baby) or backward (e.g., baby-stork) direction. The critical new finding was that both attended positive and ignored negative semantic priming occurred only for prime-target pairs presented in the forward direction. The results support either (1) a spreading inhibition model in which items associated with an ignored distractor are inhibited during prime selection or (2) a version of episodic retrieval theory in which the prime distractor and items associated with it are tagged as "to-be-ignored" during prime selection.
\end{abstract}

Recently attended objects are often responded to more quickly than new objects. This phenomenon, known as repetition or identity priming, occurs across a wide variety of tasks (Durso \& Johnson, 1979; Kolers \& Ostry, 1974; Scarborough, Cortese, \& Scarborough, 1977; see Forster, 1998; Tenpenny, 1995, for reviews). Although one's attending to a stimulus often benefits later responding to that stimulus, one's actively ignoring a stimulus can instead impair subsequent responding to that same stimulus, an effect called identity negative priming (for reviews, see Fox, 1995; May, Kane, \& Hasher, 1995; Neill, Valdes, \& Terry, 1995).

Attention to a stimulus also facilitates subsequent processing of semantically related items. Like identity priming, this positive semantic priming effect occurs across a wide variety of tasks (see Lucas, 2000; McNamara \& Holbrook, in press; Neely, 1991; for reviews). Recent experiments have also suggested that the "detrimental" effect of one's recently ignoring an item also generalizes to semantically similar items, producing semantic negative priming. However, unlike semantic positive priming, semantic negative priming has been obtained only under limited conditions and often reverses to positive priming with minor procedural changes (Fuentes \& Tudela, 1992; Ortells, Abad, Noguera, \& Lupiáñez, 2001; Ortells \& Tudela, 1996; Yee, 1991).

This research was conducted in partial fulfillment of the requirements for the degree of doctor of philosophy at the State University of New York, Albany, December 2001. I thank the chair, Jim Neely, as well as the other members of the dissertation committee, Tram Neill and Jeanette Altarriba, for their many thoughtful suggestions. I also thank David Balota, Bruce Milliken, and Paloma Marí-Beffa for their helpful comments on an earlier version of this article. Correspondence concerning this article should be addressed to K. A. Hutchison, Department of Psychology, Box 1125, Washington University, St. Louis, MO 63130 (e-mail: khutch@artsci.wustl.edu).

\section{Semantic Positive Priming}

The semantic positive priming (PP) effect was discovered by Meyer and his colleagues (Meyer \& Schvaneveldt, 1971; Meyer, Schvaneveldt, \& Ruddy, 1975) by using a lexical decision task (LDT). They found that people are faster to respond that a target letter string (e.g., cat) is a word when it is paired with a semantically related prime (e.g., $d o g$ ) rather than with an unrelated prime (e.g., table). This semantic PP effect has shown itself to be quite robust. (See Neely, 1991, for a review.)

Prospective priming. Many authors have interpreted semantic PP as evidence that the presentation of a prime automatically activates its abstract semantic memory representation and that this activation spreads to the representations of semantically related "neighbors," thereby facilitating their processing (Anderson, 1983; Collins \& Loftus, 1975; Neely, 1977; Posner \& Snyder, 1975). In addition to this automatic priming mechanism, Posner and Snyder proposed that semantic PP is also produced by a second, slower acting, limited-capacity strategic mechanism via which people use the prime to generate an expectancy for semantically related targets. There is now considerable support for the existence of both conscious expectancy and automatic semantic activation processes (de Groot, 1984; den Heyer, Briand, \& Dannenbring, 1983; Hutchison, Neely, \& Johnson, 2001; Neely, 1977; Tweedy, Lapinski, \& Schvaneveldt, 1977; see Neely, 1991, for a review). As noted by Neely, Keefe, and Ross (1989), these mechanisms are "prospective" in that they are initiated prior to the onset of the target.

Retrospective priming. Several researchers have suggested that the standard LDT involves a third strategic semantic-matching process that does not operate in other tasks such as pronunciation (Balota \& Lorch, 1986; Chiarello, Senehi, \& Nuding, 1987; Forster, 1981; Neely, 1977, 1991; Neely \& Keefe, 1989; Neely et al., 1989). 
Whereas expectancy operates in a prospective manner, increasing lexical access for a related target, semantic matching works retrospectively, taking place after lexical access has occurred for the target but before the decision/ response has been completed. According to Neely and Keefe (1989), strategically "checking back" to see whether a target is related to the preceding prime word is usually an effective strategy in the LDT because if indeed the pair is related, the target is necessarily a word (unless an experimenter were to include "related" word-nonword pairs such as boy-girk, which is typically not done). Therefore, if the pair is related, the participant is biased to respond that the target is a word, facilitating responses to word targets. However, if the pair is unrelated and the target is likely to be a nonword, the participant is biased to respond that the target is a nonword, facilitating responses to nonword targets and inhibiting responses to unrelated word targets. This semantic-matching strategy presumably does not occur in pronunciation, because knowledge that a target is related to its prime does not provide much information concerning which particular articulatory response to execute.

Some support for a retrospective-checking mechanism that operates only in the LDT comes from studies in which items are used that are "asymmetrically related," being associated in one direction but not in the other (e.g., stork-baby). For example, when given stork in a free-association task, people are likely to generate the associate baby. However, when given baby, people are highly unlikely to generate the word stork as an associate. Koriat (1981) utilized the standard LDT and obtained equal semantic PP for forward pairs (e.g., storkbaby) and backward pairs (e.g., baby-stork), relative to their respective unrelated baseline conditions (e.g., fence-baby and tree-stork). ${ }^{1}$ This finding has been replicated by several researchers using the standard LDT when the prime-to-target stimulus-onset asynchrony (SOA) is $500 \mathrm{msec}$ or greater. (See Kahan, Neely, \& Forsythe, 1999, for a review.) However, with similar SOAs in a pronunciation task that yields forward semantic PP, backward PP does not occur (see Kahan et al., 1999). Nor does it occur in a continuous $L D T$ procedure in which participants respond to both prime and target stimuli (Shelton \& Martin, 1992). McNamara and Altarriba (1988) have argued that, like pronunciation, this continuoustarget procedure eliminates semantic matching. It does so because both word and nonword targets can follow either words or nonwords. Thus, the failure to detect a semantic relation between the current and previous letter string no longer provides useful information regarding the lexicality of the current letter string. ${ }^{2}$

\section{Semantic Negative Priming}

Tipper (1985) and Tipper and Driver (1988) observed that negative priming (NP) could generalize between items belonging to the same semantic category (e.g., chair-table). They explained this effect by appealing to a spreading inhibition process analogous to the auto- matic spreading activation suggested to underlie semantic PP from attended items (Anderson, 1983; Collins \& Loftus, 1975; Neely, 1977; Posner \& Snyder, 1975). According to Tipper and Driver, when an object is ignored, inhibition beginning at the central representation of that item spreads to related items, lowering their activation levels below baseline (see Houghton \& Tipper, 1994; Tipper, Weaver, \& Houghton, 1994, for more recent models of inhibitory processes in selective attention).

Semantic NP can also be accounted for by an episodic retrieval process (Milliken, Joordens, Merikle, \& Seiffert, 1998; Neill \& Valdes, 1992; Neill, Valdes, Terry, \& Gorfein, 1992) that does not produce inhibition of a distractor item's central representation, but rather produces interference via the retrieval of the prior episode in which the distractor was ignored. According to Neill and colleagues (Neill, 1997; Neill \& Valdes, 1992; Neill et al., 1992), during a trial, an episodic trace is encoded into memory that contains information regarding how the items in the prime display were interpreted and/or responded to. This trace includes information regarding whether distractor items were ignored. When a target stimulus is later encountered in a context similar to that in which it was previously encoded as ignored, if this prior episode is retrieved before the algorithmic identification and response processes for that target stimulus run to completion, then the retrieved "to-be-ignored" tag interferes with the current response. ${ }^{3}$ Thus, NP is produced not via inhibition but rather via response competition produced by the retrieval of a past episode in which the current target was associated with a do-not-respond tag.

Neill (1997) argued that the "current target stimulus cues the retrieval of past processing episodes involving similar stimuli" (p. 1293). Accordingly, when the retrieved episode includes information about the response and/or relevance of that stimulus, semantic PP would occur if the previously encoded item semantically related to the current target had been attended, whereas NP would occur if that item had been ignored.

Variables influencing semantic NP. Unfortunately, there have been few tests of the different theories for $s e$ mantic NP because the effect itself has been so elusive, especially when words rather than pictures are used as stimuli. For instance, although Tipper and Driver (1988) found semantic NP from pictures to other pictures and from pictures to words, they failed to find an effect from words to other words. This "null" semantic NP effect for words is not uncommon. Although most published studies on semantic NP have observed this effect in at least one condition, every one of these studies has found either null effects or semantic PP in other, highly similar, conditions (Fox, 1996; Fuentes \& Tudela, 1992; MaríBeffa, Fuentes, Catena, \& Houghton, 2000; Ortells et al., 2001; Ortells \& Tudela, 1996; Richards, 1999; Yee, 1991).

In several of the studies listed above, post hoc appeals were made to methodological factors that might explain the differences in positive and negative semantic priming 
across conditions. For instance, in a cross-language semantic NP study, Fox (1996) found semantic NP only when the prime distractor was in a bilingual's dominant language and the associated probe target was in the bilingual's second language. Also, Ortells and Tudela (1996) found semantic NP only when participants were explicitly told both to attend to the cued prime and to ignore the uncued prime. However, these factors cannot account for differences in semantic NP across other studies, because these other studies have all presented the distractor (and the target) in the participant's dominant language and have all instructed people to ignore the distractors.

Yee (1991) and Ortells et al. (2001) both emphasized the importance of the SOA between the prime and probe displays, finding significant semantic NP at an SOA of $600 \mathrm{msec}$, but instead finding semantic PP at slightly longer or shorter SOAs. To explain her pattern of results, Yee argued that ignored information initially goes through a period of facilitation followed by a period of suppression. Ortells et al. later added that not only does inhibition take time to accrue, but also rapidly dissipates. There is little evidence, however, that an SOA of around $600 \mathrm{msec}$ is either necessary or sufficient for producing semantic NP. For instance, some studies have either (1) failed to obtain semantic NP under some conditions with an SOA of approximately $600 \mathrm{msec}$ (Fuentes \& Tudela, 1992; Ortells \& Tudela, 1996; Richards, 1999), or instead (2) obtained semantic NP with an SOA of greater than 1,000 msec (Marí-Beffa et al., 2000; Richards, 1999).

In addition to SOA, Richards (1999) discussed the importance of the type of probe task in obtaining semantic NP. Richards found null semantic NP in a pronunciation task but significant NP effects in a semantic categorization task, reasoning that NP effects are larger in tasks that rely more heavily on semantic-level information. However, because several studies have also obtained semantic NP with an LDT (Fox, 1996; Fuentes \& Tudela, 1992; Marí-Beffa et al., 2000; Ortells et al., 2001; Ortells \& Tudela, 1996; Yee, 1991), perhaps instead it is the forced-choice probe decision in these tasks that is critical for obtaining semantic NP. Unfortunately, these two possibilities remain unclear as well because Tipper and Driver (1988) and Chiappe and MacLeod (1995) both failed to find semantic NP for word stimuli, despite their use of a categorization task similar to the one used by Richards.

Still another factor that might be critical in determining both semantic PP and NP is the strength with which prime-target items are associated. Consistent with many theories of priming (Anderson, 1983; Collins \& Loftus, 1975; Deese, 1965), semantic PP is often much larger if the stimuli are strongly associated (see Lucas, 2000; McNamara \& Holbrook, in press, for recent discussions of the importance of association strength to semantic PP). Perhaps semantic NP is sensitive to such association as well. Indeed, Ortells et al. (2001) mentioned in a footnote that they were only able to obtain semantic NP when the stimuli were strongly associated. If, as predicted, semantic NP depends on the degree of association between the prime and the target, one should observe NP only from strongly associated stimuli.

Summary of semantic NP. Although such factors as instructions, language dominance, and prime-probe SOA have been shown to influence semantic NP in one or two studies, none of these factors aids in discriminating significant from null semantic NP across different labs and tasks. There may be some importance of task and association strength. However, because only a couple of studies have used the pronunciation task or nonassociated items, the necessity of semantic processing tasks and strong associates is presently unclear.

\section{The Present Investigation}

The present study was designed to provide a further demonstration of semantic NP while investigating a critical new question: Does semantic NP occur primarily in a prospective or retrospective manner? Inhibition theories assume that the activation level of an ignored item's semantic representation gets inhibited and that this inhibition spreads in such a way as to lower the activation level of representations of related items (Houghton \& Tipper, 1994; Tipper \& Driver, 1988). This inhibition occurs in a "prospective" manner, starting from the onset of the prime.

By contrast, Neill and colleagues (Neill, 1997; Neill \& Valdes, 1992; Neill et al., 1992) have never specified exactly how semantically associated items might produce interference in their model. As a result, there are (at least) two possible versions of episodic retrieval that could account for semantic NP. According to both versions, during the prime trial, activation spreads from the prime target and distractor to all related items increasing the activation levels above baseline. (See Neill \& Joordens, 2002.) If one of these recently activated items is then presented as a probe target, the residual activation should facilitate its identification, regardless of whether this item was activated by the prime target or the prime distractor. Therefore, both versions of the model predict prospective semantic PP due to residual activation from the prime trial. However, these models differ in terms of exactly when semantic relations between the prime and probe items produce NP.

In what I shall call the identity-tagging/associativeretrieval model, episodic tags are placed only on the target and distractor themselves during prime selection and not on any associatively related items. During the later probe trial, however, the presentation of the probe target cues past processing episodes involving not only the probe target itself, but also related items. Semantic PP occurs if the probe target is related to the attended prime target (because the response tag for the related target in the previous prime trial is the correct response to the current target on the probe trial), and semantic NP occurs if the probe target is related to the prime distractor (because the tobe-ignored tag for the related distractor in the previous 
prime trial conflicts with the correct response to the current target on the probe trial). This identity-tagging/ associative-retrieval model therefore emphasizes the importance of a retrospective associative retrieval process that occurs prior to the probe response in producing semantic PP and NP.

In contrast, an alternative model stresses the importance of "associative" activation during prime trial selection. According to this associative-tagging/identityretrieval model, during selection on the prime trial, tags are placed not only on the target and distractor themselves, but also on items activated by the target and distractor. For items activated by the target, these tags contain the response given to the target stimulus, whereas items activated by the distractor are tagged as to-be-ignored, since these items might also interfere with correct responding. During the probe trial, the presentation of the probe target cues the retrieval of episodes involving only that specific target. For items related to the prime target, PP should occur because of (1) residual activation from the prime and (2) the episodic retrieval of the prime trial response. In contrast, for items related to the prime distractor, the persisting activation from the prime trial should cause PP, whereas retrieval of the recent to-beignored tag placed on the item should interfere with responding, causing NP. The resulting outcome (PP or NP) for items activated by the prime distractor will depend on the relative contributions of activation (which produces PP) and episodic retrieval (which produces NP). (See Neill \& Joordens, 2002, for details.) This associativetagging/identity-retrieval model therefore emphasizes the importance of the prospective tagging process that occurs prior to the onset of the probe display in producing semantic PP and NP.

The present study was designed to test whether semantic PP and NP primarily occur prospectively (via associative inhibition or tagging) or retrospectively (via associative retrieval) by using items such as stork and baby that are asymmetrically associated and by presenting these items in a modified continuous LDT in either the forward direction (e.g., from stork to baby) or the backward direction (e.g., from baby to stork). As argued by McNamara and Altarriba (1988) and Shelton and Martin (1992), the use of a continuous LDT should minimize the involvement of any strategic semantic matching process because the absence of a semantic relation no longer predicts a nonword response. However, many NP studies use a "hybrid" of standard and continuous LDT. As with the standard LDT, prime and probe trials are temporally grouped by the inclusion of an intertrial interval following probe responses. However, as with the continuous procedure, responses are required during both the prime and probe trials. It is therefore of interest whether backward semantic PP effects occur under this hybrid procedure. If this procedure successfully eliminates retrospective semantic matching, semantic PP from attended primes should only occur in the forward direction. It is also of particular interest whether semantic NP from ignored items occurs prospectively or retrospectively, since the experimental designs in prior semantic NP research have not been appropriate for answering this question.

The present procedure was also designed to eliminate the problem of task switching between the prime and probe trials seen in most previous semantic NP studies in which a LDT has been used. Previous research has revealed that people respond more slowly when required to shift from one task to another on consecutive trials than when performing the same task on both trials (see Allport, Styles, \& Hsieh, 1994). Of particular interest, such a task shift between prime and probe trials has been present in five of the six previous semantic NP studies requiring LDT on the probe trial. Fox (1996) and Yee (1991) required figure and number classification, respectively, on the prime, whereas Fuentes and Tudela (1992), Ortells and Tudela (1996), and Ortells et al. (2001) required semantic categorization on the prime trial. The reason for this task-switching procedure is most likely the need for assessing accuracy during the prime trial. For example, if both a to-be-attended red word and a to-beignored green word are presented on the prime trial for an LDT and participants respond "word," there is no way to assess whether the person correctly responded to the red to-be-attended word rather than to the green to-beignored word. In the only LDT experiment to eliminate the problem of task shifting, Marí-Beffa et al. (2000, Experiment 1) found semantic NP by using a hybrid LDT similar to that in the present study, in which participants were presented with a word or nonword target at fixation flanked above and below by a to-be-ignored distractor word. However, as noted above, there is no way of knowing on a trial-by-trial basis whether their participants were following instructions and responding to the word target, or were instead responding to the to-be-ignored distractor word. This problem was reduced in the present experiment by presenting the to-be-ignored word distractors alongside to-be-attended nonword targets during the prime trial, thus eliminating the problem of task shifting while also allowing for an assessment of whether the participants were indeed responding to the correct to-beattended target stimuli.

Inhibition and episodic retrieval predictions. According to the inhibition theory of semantic NP, when selecting an appropriate response, one inhibits the representation of the potentially interfering distractors. During selection, this inhibition spreads to the representations of all associates activated by the distractor. Thus, as is shown in Figure 1, inhibition theories predict semantic NP only when the prime and probe are associated in the forward direction (e.g., stork-baby) because the inhibition applied to stork should spread to the associated item baby, but not in the backward direction (e.g., baby-stork) because the inhibition applied to baby should not spread to the nonassociated item stork.

In contrast, the identity-tagging/associative-retrieval model predicts that only the distractor item itself is tagged do-not-respond during selection, and during the probe trial, the past processing episode involving this related tagged stimulus is retrieved. Therefore, the identity-tagging/ 


\section{Ignored Prime (Forward) Ignored Prime (Backward)}

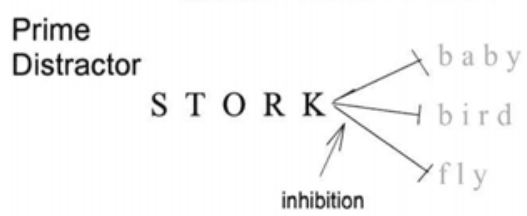

Probe

Target

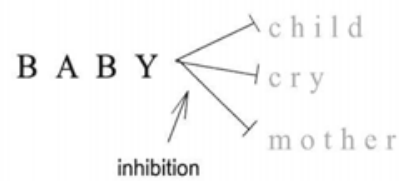

S T O R K

no effect

Prediction: NP

Figure 1. Predictions from a spreading inhibition model.

associative-retrieval model predicts interference only when asymmetrical pairs are presented in the backward direction. However, this model predicts facilitation owing to residual activation when items are presented in the forward direction (e.g., stork-baby). Therefore, as is shown in Figure 2, the identity-tagging/associative-retrievalmodel predicts that PP should occur from ignored items presented in the forward direction, whereas NP should occur from ignored items presented in the backward direction.

Similar to the inhibition model, the associative-tagging/ identity-retrieval model can predict associative NP when items are presented only in the forward direction. This model is shown in Figure 3. Notice in this figure that when an asymmetrically associated pair such as stork-baby is presented in the forward direction, the item baby is activated by the prime distractor stork and tagged do-not-respond during selection. During the later presentation of baby as a target, facilitation occurs owing to residual activation from the prime trial while the re- sponse process is slowed because the probe target baby cues the episodic retrieval of baby during the prime trial, and the retrieved do-not-respond tag interferes with the appropriate current response. However, when presented in the backward direction (e.g., baby-stork), stork is not activated by the prime distractor baby and hence does not receive a do-not-respond tag. As a result, there is neither facilitation nor interference during the later presentation of stork as a target.

These models make similar predictions in regard to priming from attended targets. Because the inhibition model involves only activation or inhibition mechanisms that supposedly act in a prospective manner, it predicts semantic PP only when pairs are presented in the forward direction, owing to residual activation and no effect from pairs presented in the backward direction. Similarly, the identity-tagging/associative-retrieval model predicts that activation spreads from the prime target to related items, producing facilitation for these items when

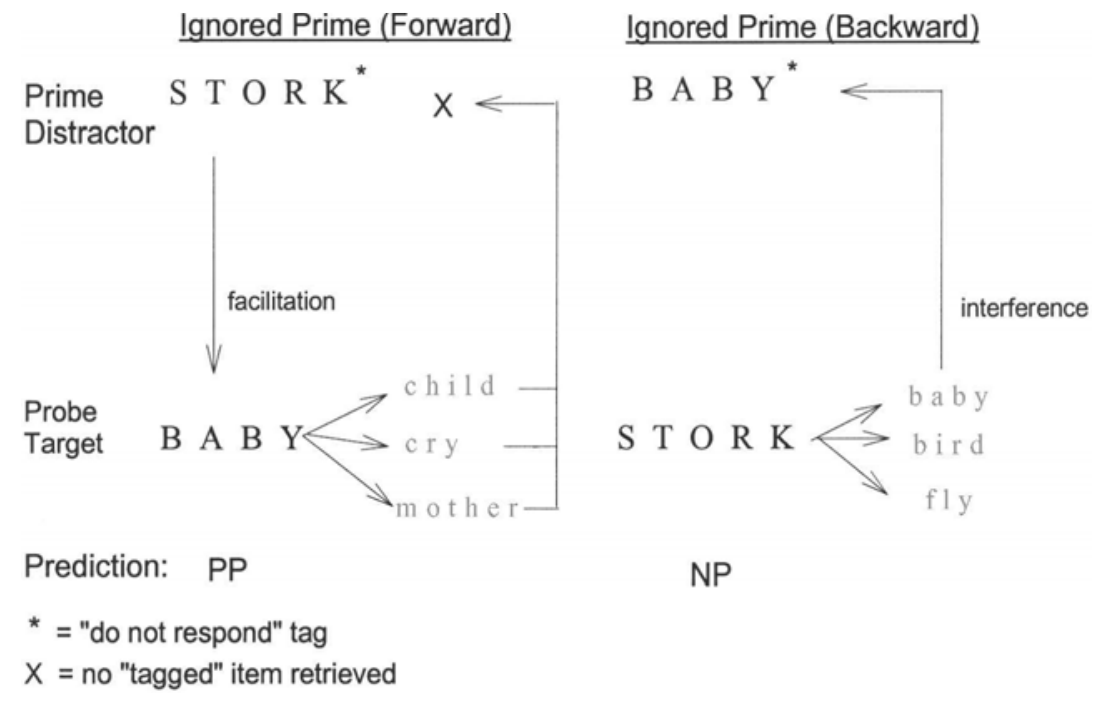

Figure 2. Predictions from the identity-tagging/associative-retrieval model. 
Ignored Prime (Forward)

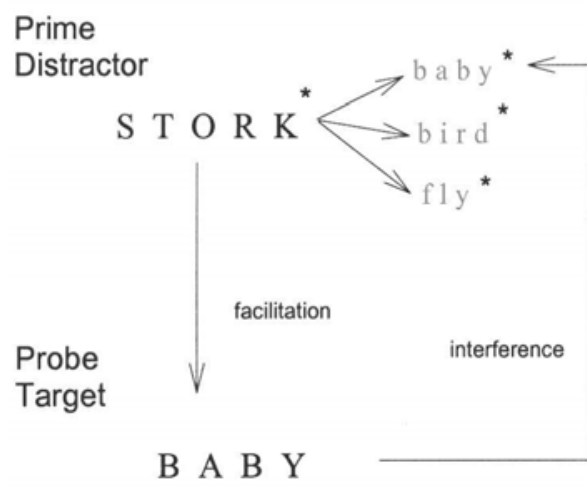

Prediction: ?

$*=$ "do not respond" tag

$\mathrm{X}=$ no "tagged" items retrieved
Ignored Prime (Backward)

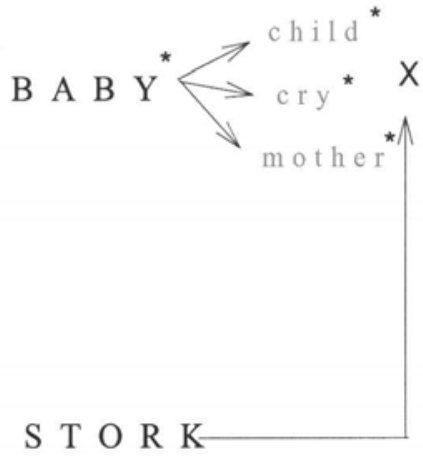

no effect

Figure 3. Predictions from the associative-tagging/identity-retrieval model.

presented in the forward direction. However, this model predicts that backward targets (e.g., stork) should cue the retrieval of the related prime target (e.g., baby), facilitating the response, whereas forward targets (e.g., baby) should function as ineffective cues and thus have no effect on the response process. Thus, this model predicts PP for forward items due to the residual activation, and PP for backward items due to episodic retrieval of the response given to the related item. The associative-tagging/ identity-retrieval model also predicts that both residual activation and episodic retrieval contribute to PP from attended items presented in the forward direction. For backward items, because they do not become activated during the prime trial, there will be no residual activation of these items, nor will there be any response tags placed on these items. Therefore, the model predicts no effect from these items.

To summarize these predictions, the inhibition and associative-tagging/identity-retrieval models predict no backward PP or NP, whereas the identity-tagging/ associative-retrieval model predicts backward PP from attended items and backward NP from ignored items. The inhibition and associative-tagging/identity-retrieval models predict forward NP from ignored items, whereas the identity-tagging/associative-retrieval model predicts $P P$ from these items. Finally, all three models predict PP from attended items presented in the forward direction.

\section{METHOD}

\section{Design}

Each prime and probe trial consisted of both a word (e.g., baby) and a nonword (e.g., luwn) presented in a spatially overlapping vertical display. On each trial, the participants attended and responded to "red" target items while ignoring "green" distractor items. In half of the trials, the word was written in red and the nonword distrac- tor was written in green, whereas in the other half of the trials, the colors were reversed. Critical trials were those in which the probe word was written in red and hence required a word response. On half of the critical trials, the prime word was attended (i.e., printed in red ink), and on the other half of the trials the prime word was ignored (i.e., printed in green ink). In addition, the prime-probe word pairs were presented in either the forward direction (e.g., stork-baby) or the backward direction (e.g., baby-stork) and were either related or unrelated. This 2 (attention) $\times 2$ (direction) $\times 2$ (prime relatedness) within-subjects design produced eight conditions: forward attended related (e.g., attend stork, respond "baby"), forward attended unrelated (e.g., attend puppet, respond "baby"), forward ignored related (e.g., ignore stork, respond "baby"), forward ignored unrelated (e.g., ignore puppet, respond "baby"), backward attended related (e.g., attend baby, respond "stork"), backward attended unrelated (e.g., attend string, respond "stork"), backward ignored related (e.g., ignore baby, respond "stork"), and backward ignored unrelated (e.g., ignore string, respond "stork"). The four unrelated priming conditions listed above were used to assess priming in the corresponding related priming conditions.

\section{Participants}

One hundred sixty-four male and female SUNY Albany undergraduates participated for partial completion of a research requirement for an introductory psychology class. All were native English speakers with normal or corrected-to-normal vision. Data from 4 participants who had overall error rates greater than $40 \%$ in either the word or nonword trials were excluded from the analysis, leaving data from a total of 160 participants.

\section{Stimuli}

The data are reported from 40 critical asymmetrically associated noncompound word pairs (e.g., dentist-pain) taken from Kahan et al. (1999), Peterson and Simpson (1989), and Thompson-Schill, Kurtz, and Gabrieli (1998). The University of South Florida Word Association Norms (Nelson, McEvoy, \& Schreiber, 1989) were used to select word pairs from these studies that contained a strong forward association (mean forward association $=.26$ ) yet little to no backward association (mean backward association $=.01$ ). Owing to the difficulty in selecting a large number of such asymmetric pairs, only five critical items appeared in each condition for each partic- 
ipant. By necessity, the critical targets on forward trials were different from those on backward trials, and these forward targets had a higher Kučera and Francis (1967) printed word frequency (mean = 113) than did the backward targets (mean $=25$ ). Therefore, to control for any item differences' contributing to priming effects, separate unrelated baselines were used to assess priming in the forward and backward conditions with priming for forward targets being measured relative to the same forward targets in the unrelated condition (counterbalancing across subjects) and priming from backward targets being measured relative to the same backward targets in the unrelated condition (counterbalancing across subjects).

The target and distractor stimuli were each approximately $0.6 \mathrm{~cm}$ high and $2.5 \mathrm{~cm}$ wide. In addition, the center of the distractor stimulus was displaced $0.8^{\circ}$ above or below the target. Each stimulus word in both the prime and probe displays was presented with an accompanying nonword matched in letter length. This was done in order to assess whether the participants were indeed responding to the correct "red" stimulus on each trial and not to the incorrect "green" stimulus that they were to ignore.

Half of the critical word probe target trials were preceded by an attended word prime (and an ignored nonword), and half were preceded by an attended nonword prime (and an ignored word). Therefore, the ignored word condition necessarily involved a response switch from a nonword response to a word response, whereas the attended repetition condition maintained the repetition of a word response. On the basis of past research showing that word responses are faster following previous word responses than following previous nonword responses (McKoon \& Ratcliff, 1995; McNamara, 1994), it was predicted that a main effect of attention would be obtained, with faster responding following attended primes. To control for this and other possible confounds (e.g., relative spatial position and/or retinal eccentricity) between the attended and ignored stimuli, separate unrelated baselines were used to measure priming in each condition.

Eight lists were created in order to counterbalance the critical word pairs across the eight experimental conditions with direction, attention, and prime relatedness as variables. Each participant saw each prime and probe word only once during the experiment. Twenty participants were tested in each of the eight lists. A list of these critical items is in the Appendix.

Forty filler trials were selected from the same sources as the critical items. These filler items were asymmetrical compound words (e.g., lip-stick) and were counterbalanced across the same eight attention $\times$ direction $\times$ prime relatedness conditions as the critical noncompound stimuli. These items were selected as fillers because, although they form compound words when presented in the forward direction, they are not strongly associated in either the forward (mean forward association $=.07$ ) or the backward (mean backward association $=.01$ ) direction. As with the critical noncompound stimuli, the compound targets on forward trials had a higher Kučera and Francis $(1967)$ printed word frequency $($ mean $=144)$ than did the backward targets $($ mean $=65)$.

In addition to the 80 word probe target trials, 80 nonword probe target trials were created. As with the word probe target trials, half of the nonword probe target trials were preceded by an attended word prime (and an ignored nonword), and half were preceded by an attended nonword prime (and an ignored word). In addition, the word distractor on these nonword probe target trials was associatively related to the word stimulus in the prime display on half of the trials in order to prevent the participants from engaging in a type of global semantic matching in which they respond "word" or "nonword" to probe targets merely by detecting a relation between any words in the two displays. Such a strategy would be ineffective in the present study because, as was so for the word target probe trials, half of the nonword target probe trials contained a word that was related to the previous prime word.

The 160 test trials were divided into four blocks of 40 trials each. Prior to receiving the experimental trials, each person received 16 practice trials. The proportions of each condition in the practice trials were equal to those of the following critical test list.

\section{Procedure}

Each individually tested participant, seated approximately $60 \mathrm{~cm}$ away from a VGA monitor, read a set of task instructions displayed on the monitor and then heard them paraphrased by the experimenter. The target stimuli were always presented centered on the display monitor with the to-be-ignored distractor displaced slightly above or below the target. Thus, attended prime targets appeared in the same position on the computer screen as the probe target, whereas ignored prime distractors appeared in a position on the computer screen other than that occupied by the probe target. However, because priming for targets presented in the attended related and ignored related conditions was measured relative to the same targets presented in either attended unrelated or ignored unrelated conditions, this confound of same versus different spatial location with attended versus ignored prime is equated for the related and unrelated conditions used to compute the priming effects. Prior to each prime trial, the participants were cued with a 700-msec fixation point $(*)$. The immediately following prime display consisted of spatially overlapping word and nonword stimuli similar to the display used by Milliken et al. (1998). The participants were instructed to press the "/" key with their right index finger if the red target item was a real English word and the "z" key with their left index finger if the red target item was a nonword. (However, see the Discussion section for a possible alternative response strategy.) Each prime display was presented for $2,500 \mathrm{msec}$ or until a response was given. After a response was made to the prime display, the participants were presented with another fixation point (*) for $700 \mathrm{msec}$. The probe display was then presented, and the participants once again made a lexical decision response to the "red" item. After a response was given (or 2,500 msec had elapsed) a 2,000-msec blank screen was displayed until a new trial began. The participants were instructed to respond to the "red" item while ignoring the "green" distractor. The participants were asked to make their responses as quickly and accurately as possible on all experimental trials. Selfpaced rest breaks were given every 40 trials.

\section{RESULTS}

Only probe trials following correct prime responses were selected for analysis. This led to the elimination of $7.5 \%$ of the data. In addition, for each participant, a geometric mean reaction time (RT) for correct word or nonword responses on probe trials was calculated for each of the conditions. Because only correct probe trials were considered for the RT analysis, an additional $6.9 \%$ of the trials preceded by correct prime responses were eliminated from the RT analysis. Group arithmetic means based on individual participants' geometric mean RTs are presented in Table 1 along with the percentage of errors. Priming effects were computed by subtracting the geometric mean RT or percent errors in a related condition from the geometric mean RT or percent errors in its corresponding unrelated condition. ${ }^{4}$

RTs and percent errors for the eight critical word conditions were submitted to analyses of variance (ANOVAs) with attention, direction, and prime relatedness all varied within subjects. The three-way attention $\times$ direction $X$ prime relatedness interaction was significant $[F(1,159)=$ 8.07, $\left.M S_{\mathrm{e}}=14,323\right]$. Because the primary interest was in the different patterns of semantic PP and NP obtained 
Table 1

Mean Reaction Times (RTs, in Milliseconds), Percent Errors, and Priming Effects for Forward, Backward, and Nonword Probe Targets Following Attended or Ignored Prime Words

\begin{tabular}{|c|c|c|c|c|c|c|c|c|}
\hline \multirow[b]{2}{*}{ Targets } & \multicolumn{4}{|c|}{ Attended Prime Worda } & \multicolumn{4}{|c|}{ Ignored Prime Word ${ }^{b}$} \\
\hline & RT & $S E$ & PE & $S E$ & $\mathrm{RT}$ & $S E$ & $\mathrm{PE}$ & $S E$ \\
\hline \multicolumn{9}{|c|}{ Forward probe targets } \\
\hline Unrelated & 737 & 12.5 & 5.2 & 0.8 & 750 & 11.2 & 4.5 & 0.8 \\
\hline Related & 700 & 11.1 & 3.6 & 0.7 & 785 & 14.7 & 2.8 & 0.6 \\
\hline Priming & $+36^{*}$ & 12.9 & 1.6 & 1.1 & $-35^{*}$ & 13.7 & 1.7 & 1.0 \\
\hline \multicolumn{9}{|c|}{ Backward probe targets } \\
\hline Unrelated & 793 & 13.2 & 8.3 & 1.1 & 831 & 13.6 & 6.9 & 1.0 \\
\hline Related & 797 & 12.5 & 6.3 & 0.9 & 831 & 16.2 & 7.6 & 1.0 \\
\hline Priming & -4 & 12.7 & 2.0 & 1.3 & 0 & 14.2 & -0.7 & 1.3 \\
\hline \multicolumn{9}{|c|}{ Nonword probe targets } \\
\hline Unrelated & 919 & 13.4 & 6.7 & 0.7 & 953 & 14.9 & 10.8 & 0.8 \\
\hline Related & 922 & 13.6 & 6.9 & 0.6 & 955 & 14.6 & 10.5 & 0.8 \\
\hline Priming & -3 & 6.6 & -0.2 & 0.6 & -2 & 8.0 & 0.3 & 0.8 \\
\hline
\end{tabular}

Note-Priming = unrelated minus related difference scores. $\quad$ aresponded to a "word" prime. ${ }^{\text {bresponded }}$ to a "nonword" prime. $\quad * p<.05$.

from attended and ignored primes as a function of presentation direction, separate ANOVAs with direction and prime relatedness as variables were done for attended and for ignored primes. For attended primes, the direction $X$ prime relatedness interaction was significant $[F(1,159)=$ $\left.4.67, M S_{\mathrm{e}}=56,151\right]$. There was $36 \pm 26 \mathrm{msec}$ of PP when primes were presented in the forward direction, but a nonsignificant $-4 \pm 25 \mathrm{msec}$ priming effect when presented in the backward direction. (When reporting an $X$ $\pm Y$ msec effect, $Y$ refers to the $95 \%$ confidence interval.) Similarly, for ignored primes, the direction $\times$ prime relatedness interaction was significant $[F(1,159)=3.97$, $\left.M S_{\mathrm{e}}=50,926\right]$. There was $35 \pm 27 \mathrm{msec}$ of NP when presented in the forward direction, but a nonsignificant $0 \pm 28 \mathrm{msec}$ of priming when presented in the backward direction.

An items analysis confirmed the pattern of priming effects reported for participants. The three-way attention $X$ direction $X$ prime relatedness interaction was significant $\left[F(1,39)=5.61, M S_{\mathrm{e}}=3,730\right]$. For attended primes, there was a marginally significant $+28 \pm 28 \mathrm{msec}$ of priming $(p=.06)$ when presented in the forward direction, but there was a nonsignificant $-2 \pm 26 \mathrm{msec}$ priming effect when primes were presented in the backward direction. For ignored primes, there was $-28 \pm 23 \mathrm{msec}$ of priming when primes were presented in the forward direction, but there was a nonsignificant $+8 \pm 27 \mathrm{msec}$ of priming when primes were presented in the backward direction.

The overall mean error rate for critical probe words following correct decisions on the prime trial was 5.9\%. An ANOVA on the error rates with attention, direction, and prime relatedness as variables showed a main effect of prime relatedness with participants making $1.2 \pm 1 \%$ fewer errors following related than unrelated primes $\left[F(1,159)=4.68, M S_{\mathrm{e}}=91.8\right]$. However, unlike the RT data, none of the higher order interactions approached significance (all $F_{\mathrm{s}}<1.5$ ).

Two two-way ANOVAs were used to examine the effects of attention and prime relatedness on RTs and er- rors on nonword probe targets. There was no effect of prime relatedness in either RTs or errors (both $F_{\mathrm{S}}<1$ ), and the effect of attention did not interact with prime relatedness (both $F \mathrm{~s}<1$ ). These findings suggest that the associative relation between a previous prime word and a current probe distractor word had no effect on responses to nonword probe targets, regardless of whether the related prime word was attended (requiring a previous word response) or ignored (requiring a previous nonword response). Therefore, merely the detection of an associative relation between any items in the prime and probe displays did not influence the participants' tendency to respond "word" or "nonword."

\section{DISCUSSION}

The use of asymmetrical associates produced semantic PP and NP effects only from prime-target pairs presented in the forward direction. Although past studies in which the standard LDT has been used have obtained backward semantic PP, this effect was argued to be due to a strategic retrospective semantic matching process that does not operate (or is at least greatly reduced) when a continuousLDT is used (McNamara \& Altarriba, 1988; Shelton \& Martin, 1992). The lack of backward semantic PP in the present experiment suggests that the present hybrid continuous procedure, similar to the procedures used in most NP studies, successfully minimized strategic semantic matching and was not compromised by any possible prime-target grouping that might have occurred due to a 2-sec interval between trials. Given this evidence for the absence of strategic semantic matching, it is of importance that semantic NP also occurred only in the forward direction, suggesting that semantic $\mathrm{NP}$ does not rely on such a retrospective strategy. In contrast, the finding of semantic NP only for items presented in the forward direction supports a prospective view that the probe target itself must be inhibited or tagged during the prime trial in order for NP to occur. Furthermore, this asymmetrical NP supports the critical role of 
association strength, proposed in the introduction, in determining semantic NP. This finding is consistent with both the inhibition and the associative-tagging/identityretrieval models.

According to the spreading inhibition model, semantic PP occurs because activation spreads to related items and produces facilitation, whereas semantic NP occurs because items associated with the prime distractor become inhibited during selection. However, exactly how this inhibition occurs in these theories is still debated. Most theorists positing inhibition models have suggested a spreading inhibition mechanism that mirrors automatic spreading activation (Neumann \& DeSchepper, 1992; Ortells \& Tudela, 1996; Tipper, 1985; Tipper \& Driver, 1988). Hence, according to these theorists, during a prime display, activation spreads to the representations of items related to the target, whereas inhibition spreads to the representations of items related to the distractor.

Houghton and Tipper (1994) suggested a different manner in which inhibition might seemingly appear to "spread" to associated items. As Houghton and Tipper pointed out, spreading inhibition cannot act in a strictly analogous fashion to spreading activation because "if the links in [a] chain are inhibitory, then activation of [unit 1] will inhibit [unit 2], the effect of which will be to disinhibit [unit 3], the exact opposite of what is required" (p. 89). Houghton and Tipper argued instead that inhibition is internal to all object representations. Within an object, inhibition functions primarily as a feedback loop, working in an opponent-process fashion to offset any accidental activation the object receives. When a prime display is turned off, this reactive inhibition continues and eventually drives the representations of activated, but irrelevant, items below baseline levels. Thus, this type of an explanation can explain the present results by assuming that (1) upon prime presentation, both the target and distractor objects are initially activated and that this activation spreads from these representations to the representations of associated items and (2) during selection, those items that are relevant continue to receive further excitatory activation, whereas activated representations that are irrelevant are inhibited (see also Malley \& Strayer, 1995, for a similar activation-based suppression model).

The present results aided in the discrimination between two newly specified versions of episodic retrieval. According to the associative-tagging/identity-retrieval model, during selection, tags are placed on all items activated during the prime trial. Items activated by the prime target receive tags containing the response given to the target stimulus, whereas items activated by the distractor are tagged "to-be-ignored." During the probe trial, the presentation of the probe target cues the retrieval of episodes involving that specific target. As a result, for items activated by the prime distractor, the retrieval of the recent to-be-ignored tag during the probe trial interferes with the response process and causes semantic NP. Consistent with this model, semantic NP was observed only when the prime distractor and probe target contained a forward association so that the probe target should be among the items activated by the prime distractor.

In contrast, the present results are inconsistent with the identity-tagging/associative-retrieval model. According to this model, semantic NP occurs because probe targets remind people of recent experiences involving related stimuli. This model predicts semantic NP in the backward direction only, because an item such as stork should serve as an effective retrieval cue for $b a b y$, but not vice versa. Contrary to this model, both PP and NP occurred for items presented in a forward, rather than the backward, direction, highlighting the critical importance of activation during the prime trial in producing semantic NP. The absence of backward priming therefore suggests that the assumptions inherent in this version of episodic retrieval are incorrect.

One possible concern involves the unorthodox method in which each display contained both a word and a nonword. The purpose for this modification in procedure from the traditional LDT was necessary to maintain the same task on prime and probe trials while still allowing for the assessment of prime accuracy. However, as a result of this modification, the participants could have adopted an alternative strategy. Although all participants were instructed to attend to the red item and respond "/" if it was a word and " $z$ " if it was a nonword, the participants might have instead solved the task by first identifying the word stimulus and then responding "/" if the word was red and "z" if the word was green. However, this explanation seems implausible because it implies that information regarding the lexicality (i.e., word or nonword) of an item becomes available prior to information regarding its color.

There are two pieces of evidence that argue against the use of this alternative strategy. The strongest evidence concerns the predictions stemming from the translation of a decision (e.g., word or nonword) into a response (e.g., "/" or "z"). In general, participants should show PP on consecutive word decisions. If the participants were following instructions, this should translate into PP only in the correct corresponding "/" responses. As is shown in Table 1, this pattern was obtained for items presented in the forward direction. However, the alternative strategy predicts that there should be PP in both the wordword $(\mathrm{W}-\mathrm{W})$ conditions and the nonword-nonword (NW-NW) conditions because in both cases the word stimulus is the same color across trials. However, the difference in pattern, with PP in the forward $\mathrm{W}-\mathrm{W}$ condition and no effect in the NW-NW condition, argues against the use of this strategy. Another piece of evidence against the alternative strategy is that the present experiment replicated the ubiquitous finding in lexical decision experiments that responses are significantly faster when the target (i.e., red item) requires a word decision than when the target requires a nonword decision (see Neely, 1991, for a review). If the participants were responding only to word stimuli and responding accordingly, the 159 -msec difference in RT between words and nonwords observed in the present experiment would merely represent a handedness advantage. Although such a handed- 
ness advantage might be possible, when combined with the lack of PP in the NW-NW condition, these two findings suggest that the participants were indeed following instructions and responding only to the correct "red" target stimulus on each trial.

Another possible concern is that the ignored conditions required a response switch from a nonword response to the prime to a word response to the probe. As mentioned earlier, separate unrelated baselines were used to control for slowed word responding following a nonword response. Nonetheless, it is still possible that the effect of switching responses could interact with relatedness. For instance, the observed NP in the forward condition could be due to the retrieval of a "respond nonword" tag placed on associated items during the prime trial, rather than a to-be-ignored tag. Although this is an intriguing possibility, Marí-Beffa et al. (2000) found significant semantic NP following succeeding word responses using a similar procedure, suggesting that such a response switch is not necessary to produce semantic NP. However, whether or not such a response switch contributes to semantic NP is an interesting question that requires additional experimentation.

\section{Milliken et al. (1998) and Temporal Discriminability}

Although the present results have been interpreted in terms of Neill and colleagues' episodic retrieval theory, it should be emphasized that Milliken et al.'s (1998) temporal discrimination version of episodic retrieval can be embellished in similar ways to account for the data. Milliken et al. suggested a two-stage attentional process. In the first stage, the attentional system classifies a stimulus as new or old. Once this categorization is made, the second stage begins in which responses to old stimuli are retrieved directly from memory, and responses to new stimuli are computed through the algorithmic process. Contrary to Neill and colleagues, Milliken et al. proposed that NP occurs during the initial categorization stage, prior to memory retrieval. This occurs because distractor items have a weaker memory trace and therefore take longer to classify as old, pushing the second stage back in time. Therefore, according to Milliken et al., the key to identity NP is not any inhibition or tagging process during the prime trial, but rather the fact that distractor items are of a "moderate" familiarity, delaying their classification as either old or new. Milliken et al.'s temporal discrimination theory could account for the present results by suggesting that only items activated by the prime distractor reach this intermediate level of familiarity (e.g., stork activates baby).

\section{Episodic Retrieval and Semantic Matching}

There is some uncertainty concerning the strategic nature of semantic retrieval mechanisms. As originally stated by Logan (1988), the episodic retrieval mechanism is supposedly an automatic process, in that directing attention to a current target "automatically" cues the retrieval of past episodes involving similar stimuli. In- deed, Logan called such a retrieval process an "unavoidable consequence of attention." (p. 493). By contrast, Kane, Hasher, Rahhal, and Stolzfus (1997) argued that episodic retrieval is a strategic process that should improve when participants are made aware of related prime-target trials. Kane et al. (1997) obtained significant identity NP when including attended repetition (AR) trials, but Kane, Hasher, Stolzfus, Zacks, and Connelly (1994) failed to find any effect in the absence of these trials. Therefore, the influence of strategic processing on episodic retrieval is currently unknown.

Contrary to episodic retrieval, retrospective semantic matching is most often thought to reflect a strategic process with backward priming effects often used as markers of such a strategy (Kahan et al., 1999; Neely, 1991; Peterson \& Simpson, 1989; Seidenberg, Waters, Sanders, \& Langer, 1984; Shelton \& Martin, 1992). The absence of backward priming in the present experiment therefore suggests that the participants were not engaged in this process. If indeed the forward semantic NP obtained in the present study were due to episodic retrieval, this process must be independent from any semantic matching process. It would be of interest to vary such factors as (1) the proportion of unrelated prime-target pairs that contain nonword targets (the so-called nonword ratio), (2) the proportion of backward versus forward items, or (3) the type of task used to see how these factors might influence semantic NP. If semantic NP and backward PP are indeed independent mechanisms, one might find an increase in backward PP with no corresponding effect in semantic NP. On the other hand, if both these labels describe the same process, an increase in backward PP should be accompanied by a corresponding increase in NP. In short, more studies are needed to determine whether semantic NP could also occur under conditions that encourage the use of a retrospective associative process.

\section{An Integrative Model?}

Perhaps one of the strongest contributions of the present study is in highlighting the similarity between inhibitory and episodic retrieval theories of NP. In particular, the associative-tagging/identity-retrieval model in which items activated by the prime distractor are tagged "do not respond" is virtually indistinguishable from a reactive inhibition model in which the representations of these items become inhibited.

However, over the years, this debate between inhibition and episodic retrieval has proven fruitful, providing useful insights into the mechanisms of NP. As Tipper (2001) has argued recently, the two theories together have led to a better understanding of the "entire" selective attention process. Inhibitory theories have tended to focus on factors such as processing load or behavioral goals that influence the selection process, whereas episodic retrieval theories have focused more on factors such as contextual similarity or temporal discriminability that influence the retrieval process. In attempting to integrate the two theories, Tipper (2001) argued that in order to recognize an object, one must have access to prior processing episodes 
involving similar objects. Thus, as claimed by episodic retrieval theories, Tipper (2001) suggested that a probe target cues the retrieval of the prime's representation. Retrieval of this episode during the probe trial "reinstates" the representation's inhibitory state, causing NP. This integrated explanation of NP therefore combines the importance of both selection and retrieval processes.

If indeed this integrated model in which inhibitory states are retrieved and reinstated during the probe trial is accepted, further attempts to distinguish between the inhibition and traditional episodic retrieval theories would be fruitless, since both would now predict more NP under contextually similar conditions. As a result, the difference between tagging and inhibition of irrelevant distractors would merely reflect one's personal wording preference.

As an alternative to such an integrative model, Neill and Mathis (1998; see also MacDonald \& Joordens, 2000; Wood \& Milliken, 1998) have recently described a modified version of episodic retrieval that is less confusable with distractor inhibition theories. Similar to the more "traditional" episodic retrieval accounts, the transfer inappropriate processing (TIP) model posits that episodes contain information regarding how items in a display were processed. However, the TIP model adds the crucial assumption that the retrieval of past processing episodes involving similar stimuli reinstates comparable processing of an item in the present instance. This assumption is roughly analogous to the reinstated inhibitory state proposed by Tipper (2001), but suggests that what is reinstated (i.e., relevant features, interpretations, responses, goals, etc.) during a probe task depends on the nature of the processing done during the earlier episode. As a result, the TIP theory proposes that NP results from retrieving prime processing that is incompatible with current processing, rather than by retrieving an episode containing information that an instance had been ignored (or simply one containing no appropriate response information). This additional assumption allows the TIP theory to account for recent findings of NP from attended, as well as ignored, stimuli when the type of processing alters between prime and probe trials (MacDonald \& Joordens, 2000; MacDonald, Joordens, \& Seergobin, 1999; Wood \& Milliken, 1998).

However, because TIP theory is part of a general model intended to account for interference across a variety of experimental paradigms including NP, inhibition of return, repetition blindness, and the before-disruption effect, this theory might be too general in its present form to derive specific predictions. As noted by Neill and Mathis (1998), "rather than invoking transfer-appropriate processing (TAP) and transfer-inappropriate processing (TIP) as explanatory constructs, a more appropriate goal is to specify conditions under which repetitions are likely to facilitate performance (TAP) or hamper performance (TIP)" (p. 33).

\section{CONCLUSIONS}

The critical new finding obtained in the present study is that semantic NP occurs when items are presented in the forward direction, but not when items are presented in the backward direction. This result lends more support to the crucial role of association strength, in that prime trial activation is crucial for determining later semantic NP. As a result, this finding eliminates one possible version of episodic retrieval theory in which semantic NP occurs due to the retrieval of associated items during the probe trial. It is concluded that either a spreading inhibition model or an associative-tagging/identity-retrieval version of episodic retrieval could potentially explain the present findings, as could more recent theories such as Tipper's (2001) integrated inhibition/episodic retrieval approach and Neill and colleagues' (Neill \& Mathis, 1998; Wood \& Milliken, 1998) TIP/TAP theory.

\section{REFERENCES}

Allport, D. A., Styles, E. A., \& Hsieh, S. (1994). Shifting intentional set: Exploring the dynamic control of tasks. In C. Umiltà \& M. Moscovitch (Eds.), Attention and performance XV (pp. 421-451). Cambridge, MA: MIT Press.

Anderson, J. R. (1983). A spreading activation theory of memory. Journal of Verbal Learning \& Verbal Behavior, 22, 261-295.

Balota, D. A., \& Lorch, R. F. (1986). Depth of automatic spreading activation: Mediated priming effects in pronunciation but not in lexical decision. Journal of Experimental Psychology: Learning, Memory, \& Cognition, 12, 336-345.

Chiappe, D. L., \& MacLeod, C. M. (1995). Negative priming is not task bound: A consistent pattern across naming and categorization tasks. Psychonomic Bulletin \& Review, 2, 364-369.

Chiarello, C., Senehi, J., \& Nuding, S. (1987). Semantic priming with abstract and concrete words: Differential asymmetry may be postlexical. Brain \& Language, 31, 43-60.

Collins, A. M., \& Loftus, E. (1975). A spreading-activation theory of semantic processing. Psychological Review, 82, 407-428.

DEESE, J. (1965). The structure of associations in language and thought. Baltimore: Johns Hopkins University Press.

DE Groot, A. M. (1984). Primed lexical decision: Combined effects of the proportion of related prime-target pairs and the stimulus-onset asynchrony of prime and target. Quarterly Journal of Experimental Psychology, 36A, 253-280.

Den Heyer, K., Briand, K., \& DAnnenbring, G. L. (1983). Strategic factors in a lexical decision task: Evidence for automatic and attentiondriven processes. Memory \& Cognition, 11, 374-381.

Durso, F. T., \& Johnson, M. K. (1979). Facilitation in naming and categorizing repeated pictures and words. Journal of Experimental Psychology: Human Learning \& Memory, 5, 449-459.

Forster, K. I. (1981). Priming and the effects of sentence and lexical constraints on naming time: Evidence for autonomous lexical processing. Quarterly Journal of Experimental Psychology, 33A, 465-495.

FoRSTER, K. I. (1998). The pros and cons of masked priming. Journal of Psycholinguistic Research, 27, 203-233.

Fox, E. (1995). Negative priming from ignored distractors in visual selection: A review. Psychonomic Bulletin \& Review, 2, 145-173.

Fox, E. (1996). Cross-language priming from ignored words: Evidence for a common representational system. Journal of Memory \& Language, 35, 353-370.

Fuentes, L. J., \& Tudela, P. (1992). Semantic processing of foveally and parafoveally presented words in a lexical decision task. Quarterly Journal of Experimental Psychology, 45A, 299-322.

Houghton, G., \& Tipper, S. P. (1994). A model of the dynamics of selective attention. In D. Dagenbach \& T. H. Carr (Eds.), Inhibitory processes in attention, memory, and language (pp. 64-79). San Diego: Academic Press.

Hutchison, K. A., Neely, J. H., \& Johnson, J. D. (2001). With great expectations, can two "wrongs" prime a "right"? Journal of Experimental Psychology: Learning, Memory, \& Cognition, 27, 14511463.

Kahan, T. A., Neely, J. H., \& Forsythe, W. J. (1999). Dissociated 
backward priming effects in lexical decision and pronunciation tasks. Psychonomic Bulletin \& Review, 6, 105-110.

Kane, M. J., Hasher, L., RahHal, T., \& Stolzfus, E. R. (1997). Dual mechanisms of negative priming. Journal of Experimental Psychology: Human Perception \& Performance, 23, 632-650.

Kane, M. J., Hasher, L., Stolzfus, E. R., Zacks, R. T., \& Connelly, S. L. (1994). Inhibitory attentional mechanisms and aging. Psychology \& Aging, 9, 103-112.

Kiger, J. I., \& GLASS, A. L. (1983). The facilitation of lexical decisions by a prime occurring after the target. Memory \& Cognition, 11, 356365.

Kolers, P. A., \& Ostry, D. J. (1974). Time course of loss of information regarding pattern analyzing operations. Journal of Verbal Learning \& Verbal Behavior, 13, 599-612.

KorIAT, A. (1981). Semantic facilitation in lexical decision as a function of prime-target association. Memory \& Cognition, 9, 587-598.

KuČERA, H., \& FranCIS, W. N. (1967). Computationalanalysis of present day American English. Providence, RI: Brown University Press.

LoGAN, G. D. (1988). Toward an instance theory of automatization. Psychological Review, 95, 492-527.

LuCAS, M. (2000). Semantic priming without association: A meta-analytic review. Psychonomic Bulletin \& Review, 7, 618-630.

MacDonald, P. A., \& Joordens, S. (2000). Investigating a memorybased account of negative priming: Support for a selection-feature mismatch. Journal of Experimental Psychology: Human Perception \& Performance, 26, 1478-1496.

MacDonald, P. A., Joordens, S., \& Seergobin, K. N. (1999). Negative priming effects that are bigger than a breadbox: Attention to distractors does not eliminate negative priming, it enhances it. Memory \& Cognition, 27, 197-207.

Malley, G. B., \& Strayer, D. L. (1995). Effect of stimulus repetition on positive and negative identity priming. Perception \& Psychophysics, 57, 657-667.

Marí-Beffa, P., Fuentes, L. J., Catena, A., \& Houghton, G. (2000). Semantic priming in the prime task effect: Evidence of automatic semantic processing of distractors. Memory \& Cognition, 28, 635-647.

May, C. P., Kane, M. J., \& Hasher, L. (1995). Determinants of negative priming. Psychological Bulletin, 118, 35-54.

McKoon, G., \& RATCLIFF, R. (1995). Conceptual combinations and relational contexts in free association and in priming in lexical decision and naming. Psychonomic Bulletin \& Review, 2, 527-533.

McNamara, T. P. (1994). Theories of priming: II. Types of primes. Journal of Experimental Psychology: Learning, Memory, \& Cognition, 20, 507-520.

McNamara, T. P., \& Altarriba, J. (1988). Depth of spreading activation revisited: Semantic mediated priming occurs in lexical decisions. Journal of Memory \& Language, 27, 545-559.

McNamara, T. P., \& Holbrook, J. B. (in press). Semantic memory and priming. In I. B. Weiner (Series Ed.), A. F. Healy, \& R. Proctor (Vol. Eds.), Comprehensive handbookof psychology: Vol. 4. Experimental Psychology. New York: Wiley.

Meyer, D. E. \& Schvaneveldt, R. W. (1971). Facilitation in recognizing pairs of words: Evidence of a dependence between retrieval operations. Journal of Experimental Psychology, 90, 227-234.

Meyer, D. E., Schvaneveldt, R. W., \& Ruddy, M. G. (1975). Loci of contextual effects in visual word recognition. In P. M. A. Rabbitt \& S. Dornic (Eds.), Attention and performance V (pp. 98-118). New York: Academic Press.

Milliken, B., Joordens, S., Merikle, P. M., \& Seiffert, A. E. (1998). Selective attention: A reevaluation of the implications of negative priming. Psychological Review, 105, 203-229.

NeELY, J. H. (1977). Semantic priming and retrieval from lexical memory: Roles of inhibitionless spreading activation and limited-capacity attention. Journal of Experimental Psychology: General, 106, 226254.

Neely, J. H. (1991). Semantic priming effects in visual word recognition: A selective review of current findings and theories. In D. Besner \& G. W. Humphreys (Eds.), Basic processes in reading: Visual word recognition (pp. 264-336). Hillsdale, NJ: Erlbaum.
Neely, J. H. \& KeEfe, D. E (1989). Semantic context effects in visual word processing: A hybrid prospective/retrospective processing theory. In G. H. Bower (Ed.), The psychology of learning and motivation: Advances in research and theory (Vol. 24, pp. 207-248). New York: Academic Press.

Neely, J. H., Keefe, D. E., \& Ross, K. L. (1989). Semantic priming in the lexical decision task: Roles of prospective prime-generated expectancies and retrospective semantic matching. Journal of Memory \& Language, 15, 1003-1019.

NEILL, W. T. (1997). Episodic retrieval in negative priming and repetition priming. Journal of Experimental Psychology: Learning, Memory, \& Cognition, 23, 1291-1305.

NeILl, W. T., \& Joordens, S. (2002). Negative priming and stimulus repetition: A reply to Grison and Strayer (2001). Perception \& Psychophysics, 64, 855-860.

NeILl, W. T., \& Mathis, K. M. (1998). Transfer-inappropriate processing: Negative priming and related phenomena. In D. L. Medin (Ed.), The psychology of learning and motivation: Advances in research and theory (Vol. 38, pp. 1-44). San Diego: Academic Press.

Neill, W. T., \& VAldes, L. A. (1992). Persistence of negative priming: Steady state or decay? Journal of Experimental Psychology: Learning, Memory, \& Cognition, 18, 565-576.

Neill, W. T., Valdes, L. A., \& Terry, K. M. (1995). Selective attention and the inhibitory control of cognition. In F. N. Dempster \& C. J. Brainerd (Eds.), Interference and inhibition in cognition (pp. 207-261). San Diego: Academic Press.

Neill, W. T., Valdes, L. A., Terry, K. M., \& Gorfein, D. S. (1992). Persistence of negative priming: Evidence for episodic trace retrieval. Journal of Experimental Psychology: Learning, Memory, \& Cognition, 18, 993-1000.

Nelson, D. L., McEvoy, C. L., \& Schreiber, T. (1989). The University of South Florida word, rhyme, and word fragment norms. Unpublished manuscript.

Neumann, E., \& DeSchepper, B. G. (1992). An inhibition based fan effect: Evidence for an active suppression mechanism in selective attention. Canadian Journal of Psychology, 46, 1-40.

Ortells, J. J., Abad, M. J. F., Noguera, C., \& Lupiáñez, J. (2001). Influence of prime-probe stimulus onset asynchrony and prime precuing manipulations on semantic priming effects with words in a lexical decision task. Journal of Experimental Psychology: Human Perception \& Performance, 27, 75-91.

Ortells, J. J., \& Tudela, P. (1996). Positive and negative semantic priming of attended and unattended parafoveal words in a lexical decision task. Acta Psychologica, 94, 209-226.

Peterson, R. R. \& Simpson, G. B. (1989). The effect of backward priming on word recognition in single-word and sentence contexts. Journal of Experimental Psychology: Learning, Memory, \& Cognition, 15, 1020-1032.

Posner, M. I., \& Snyder, C. R. (1975). Attention and cognitive control. In R. L. Solso (Ed.), Information processing and cognition: The Loyola symposium (pp. 55-85). Hillsdale, NJ: Erlbaum.

RICHARDS, A. (1999). The effects of cueing target location and response mode on interference and negative priming using a visual selection paradigm. Quarterly Journal of Experimental Psychology, 52A, 449-463.

Scarborough, D. L., Cortese, C., \& Scarborough, H. (1977). Frequency and repetition effects in lexical memory. Journal of Experimental Psychology: Human Perception \& Performance, 3, 1-17.

Seidenberg, M. S., Waters, G. S., Sanders, M., \& Langer, P. (1984). Pre- and postlexical loci of contextual effects on word recognition. Memory \& Cognition, 12, 315-328.

Shelton, J. R., \& Martin, R. C. (1992). How semantic is automatic semantic priming? Journal of Experimental Psychology: Learning, Memory, \& Cognition, 18, 1191-1210.

TEnPEnNY, P. L. (1995). Abstractionist versus episodic theories of repetition priming and word identification. Psychonomic Bulletin \& Review, 2, 339-363.

Thompson-Schill, S. L., Kurtz, K. J., \& Gabrieli, J. D. E. (1998). Effects of semantic and associative relatedness on automatic priming. Journal of Memory \& Language, 38, 440-458. 
TIPPER, S. P. (1985). The negative priming effect: Inhibitory priming by ignored objects. Quarterly Journal of Experimental Psychology, 37A, 571-590.

TIPPER, S. P. (2001). Does negative priming reflect inhibitory mechanisms? A review and integration of conflicting views. Quarterly Journal of Experimental Psychology, 54A, 321-334.

Tipper, S. P., \& Driver, J. (1988). Negative priming between pictures and words in a selective attention task: Evidence for semantic processing of ignored stimuli. Memory \& Cognition, 16, 64-70.

Tipper, S. P., Weaver, B., \& Houghton, G. (1994). Behavioural goals determine inhibitory mechanisms of selective attention. Quarterly Journal of Experimental Psychology, 47A, 809-840.

Tweedy, J. R., Lapinski, R. H., \& Schvaneveldt, R. W. (1977). Semantic-context effects on word recognition: Influence of varying the proportion of items presented in an appropriate context. Memory \& Cognition, 5, 84-89.

Wood, T. J., \& Milliken, B. (1998). Negative priming without ignoring. Psychonomic Bulletin \& Review, 5, 470-475.

YEE, P. L. (1991). Semantic inhibition of ignored words during a figure classification task. Quarterly Journal of Experimental Psychology, 43A, 127-153.

\section{NOTES}

1. The term backward priming has also been used by Kiger and Glass (1983) to explain facilitation in lexical decision for a target (e.g., apple) when its forward-associated prime (e.g., fruit) briefly lags behind the target in presentation. It was suggested that the priming obtained in this task was due to the temporal overlap in processing between the prime and target. This procedure and interpretation are different from the present meaning of backward priming in which participants respond to the second item presented, and the terms forward and backward refer to the direction in which the prime-target pairs are associated.

2. Although McNamara and Altarriba (1988) argued that the lack of semantic matching in the continuous-target task is due to the fact that the target-prime relationship no longer predicts the correct response, other possible reasons may exist. For instance, one might argue that in presenting each item separately for a response, the salience of primetarget relations is reduced relative to experiments in which participants silently read a prime word to themselves while preparing to make a response to a forthcoming target.

3. Neill et al. (1992) stated that negative priming could result either from the retrieval of an explicit to-be-ignored tag from a prior episode or instead simply from the retrieval of a prior episode lacking any appropriate response information. This latter hypothesis is similar to Milliken et al.'s (1998) temporal discrimination theory of episodic retrieval addressed in the Discussion section.

4. A separate two-way ANOVA was used to test the effects of attention and direction on the four critical unrelated baseline conditions. As expected, responses were faster following attended primes than following ignored primes and for target words presented in the forward direction than in the backward direction. These differences suggest that response repetition and target frequency influenced overall RT and therefore justify the need for separate unrelated baselines for each condition to reflect the influence of prime relatedness only, and not simply effects of attention or direction per se. 


\section{APPENDIX}

Critical Prime-Target Pairs (Forward Direction)

\begin{tabular}{|c|c|}
\hline$\underline{\text { Prime Word (Prime Nonword) }}$ & Probe Word (Probe Nonword) \\
\hline 1. puppet (vodern) & string (feyond) \\
\hline 2. stork (whilp) & baby (luwn) \\
\hline 3. beaver (sostem) & $\operatorname{dam}(\operatorname{mog})$ \\
\hline 4. denim (keing) & blue (awaf) \\
\hline 5. termite (schwamp) & wood (gont) \\
\hline 6. canyon (tucket) & deep (mook) \\
\hline 7. zebra (basim) & stripes (locered) \\
\hline 8. maze (evey) & lost (poro) \\
\hline 9. duck (flal) & water (doage) \\
\hline 10. stove (hotch) & hot (oap) \\
\hline 11. chip (yome) & shoulder (wearmone) \\
\hline 12. fan (imp) & air (hof) \\
\hline 13. towel (prain) & dry (cim) \\
\hline 14. garlic (clavor) & smell (koker) \\
\hline 15. lamp (yome) & light (blude) \\
\hline 16. afraid (horrow) & run (dor) \\
\hline 17. dentist (vassive) & pain (mimp) \\
\hline 18. sheet (daist) & paper (haulf) \\
\hline 19. penguin (silonce) & tuxedo (carrel) \\
\hline 20. indian (breasp) & feather (shoupew) \\
\hline 21. piano (basim) & key (dap) \\
\hline 22. alibi (laken) & crime (nerse) \\
\hline 23. path (darp) & road (kead) \\
\hline 24. onion (mutch) & cry (tig) \\
\hline 25. interest (cylinzer) & money (owmer) \\
\hline 26. pigeon (negend) & statue (mascle) \\
\hline 27. picnic (plunet) & ant (hin) \\
\hline 28. factory (burious) & plant (himal) \\
\hline 29. bar (nop) & drink (aftep) \\
\hline 30. tobacco (shoriff) & smoke (asode) \\
\hline 31. crown (lubel) & king (smot) \\
\hline 32. mint (bany) & candy (smrat) \\
\hline 33. eagle (crayz) & bird (yake) \\
\hline 34. sleet (clopi) & snow (stel) \\
\hline 35. cottage (nemmer) & house (papre) \\
\hline 36. stem (quib) & flower (yelmar) \\
\hline 37. engine (mupack) & car (zup) \\
\hline 38. usher (yeldo) & movie (craty) \\
\hline 39. lizard (trocky) & snake (vepra) \\
\hline 40. scissors (grollend) & cut (dob) \\
\hline
\end{tabular}

(Manuscript received December 21, 2001; revision accepted for publication July 9, 2002.) 